\title{
The Relationship between Socioeconomic Level, and the Prevalence of Masked Hypertension and Asymptomatic Organ Damage
}

$\underline{\text { Ihsan Ates }}{ }^{1}$, Mustafa Altay ${ }^{1}$, Mustafa Kaplann ${ }^{1}$, Nihal Özkayar ${ }^{2}$, Güvenç Toprak ${ }^{1}$, M.Erdem Alagüney ${ }^{1}$, Adem Özkara ${ }^{3-4}$

${ }^{1}$ Ankara Numune Education and Research Hospital, Department of Internal Meicine, Ankara, Turkey

${ }^{2}$ Ankara Numune Education and Research Hospital, Department of Nephrology, Ankara, Turkey

${ }^{3}$ Ankara Numune Education and Research Hospital, Department of Family Medicine, Ankara, Turkey

${ }^{4}$ Hitit University Medical Faculty, Familiy Medicine Department, Corum, Turkey

\section{Objectives:}

\section{Methods:}

\section{Results:}

Figure 1.

\section{Conclusions:}

A significantly higher percentage of patients with MHT had AOD, as compared to those with EHT, in a low socioeconomic district of Ankara. Based on this finding, we think patients that present with hypertensive symptoms but have a normal BP should be advised to measure their BP at home. asymptomatic organ damage (AOD) in a low socioeconomic district of Ankara, Turkey. for MHT were retrospectively reviewed. The essential hypertension (EHT) included 86 patients screened for AOD. The presence of AOD in patients diagnosed with MHT and the EHT group was recorded.

Among the 712 patients, 206 were diagnosed with EHT and among the remaining 506 patients, 73 were diagnosed with MHT. The patients with MHT had a significantly higher left ventricular mass index, carotid intima media thickness, and 24-h urinary microalbuminuria level (all indicators of AOD) than those with EHT.
This study aimed to determine the prevalence of masked hypertension (MHT) and its association with

Data obtained from the medical records of 712 patients with no known diagnosis of hypertension that presented to a polyclinic due to symptoms related to elevated blood pressure (BP) and were screened

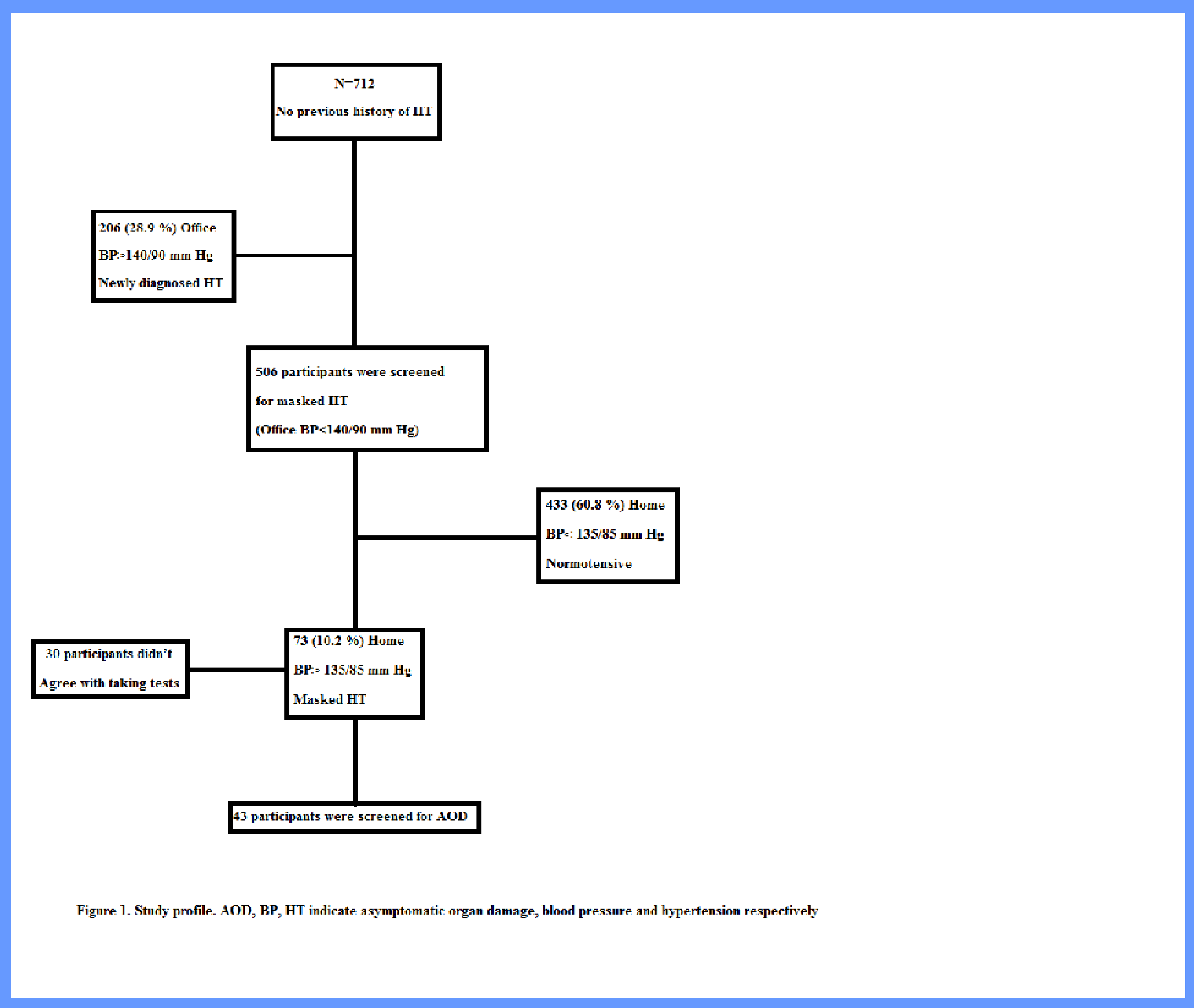

1.Pickering TG, Davidson K, Gerin W,Schwartz JE: Masked hypertension. Hypertension, 2002. 40(6): p. 795-6. 2.Mancia G, Fagard R, Narkiewicz K et al: 2013 ESH/ESC guidelines for the management of arterial hypertension: the Task Force for the Management of Arterial Hypertension of the European Society of Hypertension (ESH) and of the European Society of Cardiology (ESC). Eur Heart J, 2013. 34(28): p. $2159-219$. 